\title{
Enfoque ortodóncico en el tratamiento multidisciplinario de pacientes adultos El «stripping» y sus efectos sobre el diente y el periodonto
}

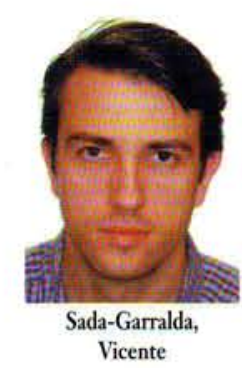

\author{
Orthodontic approach in the multidisciplinary treatment of adult patients \\ "Stripping" rechnique and its effects on tooth and periodontal lissues
}

\section{Sada-Garralda, Vicente * Caffesse, Raúl G.}

*Práctica privada dedicada en exclusiva a la ortodoncia en Navarra.

**Profesor del Postgrado de Periodoncia Universidad Autónoma de Nuevo León, Monterrey,México
Resumen: La presente comunicación relata las bases de la técnica del remodelado interproximal en el tratamiento ortodóncico aplicado a pacientes adultos con enfermedad periodontal. Se han resumido sus bases biológicas, sus ventajas, indicaciones y técnica. Los resultados logrados han sido ejemplificados con la presentación de dos casos clínicos cuyos tratamientos fueron postergados varios años por la negativa de los pacientes a aceptar las extracciones recomendadas por varios especialistas. Es evidente que en los casos indicados el tratamiento ortodóncico debe formar parte del enfoque multidisciplinario del paciente periodontal adulto con el objeto de obtener resultados funcionales y estéticos que satisfagan tanto al profesional como al paciente.

Palabras clave: Remodelado interproximal, Tratamiento ortodóncico, Enfermedad periodontal, Enfoque multidisciplinar.

\begin{abstract}
The present communication has reviewed the basic principles of the stripping technique as part of the orthodontic treatment of adult patients with periodontal disease. Its biological bases, advantages, indications and technique have been summarized. The results achieved have been documented with the presentation of two clinical cases whose treatments were postponed for several years due to the patient's negative to accept the extractions that were recommended by several specialists. It is evident that in cases where there is an indication, orthodontic treatment should be part of the multidisciplinary approach of the adult periodontal patient. This way, functional and aesthetic results that satisfy both the professional and the patient will be obtained.
\end{abstract}

Key words: Stripping, Orthodontic treatment, Periodontal disease, Multidisciplinary approach.

\begin{tabular}{ccc}
\hline $\begin{array}{c}\text { Fecha recepción } \\
07-10-2003\end{array}$ & Fecha última revisión & Fecha aceptación \\
$12-12-2003$ & $18-03-2004$ \\
\hline
\end{tabular}

BIBLID [1138-123X (2004)9:2; marzo-abril 125-256]

Sada-Garral V, Caffesse RC. Enfoque ortodóncico en el tratamiento multidisciplinario de pacientes adultos. El "stripping" y sus efectos sobre el diente y el periodontORCOE 2004;9(2):179-189. 


\section{Introducción}

En una publicación anterior, se ha enfatizado la estrecha relación que debe existir entre ortodoncia y periodoncia en el tratamiento del paciente adulto y las ventajas que eso conlleva para el resultado logrado especialmente en la solución de casos complejos. Se ha considerado el efecto que el movimiento ortodóncico ejerce en los tejidos periodontales, la importancia de la dimensión vertical así como la necesidad de coordinar adecuadamente las etapas terapeuticas periodontales y ortodóncicas'.

En este trabajo, se intenta presentar la posibilidad de utilizar el remodelado interproximal o "stripping" para obtener espacio necesario que permita alinear adecuadamente dientes en malposición, así como sus ventajas frente a otros enfoques posibles.

\section{Definición}

Se denomina "stripping" al desgaste mecánico parcial del esmalte interproximal realizado por el profesional, con el objeto de obtener espacio para la ubicación ortodóncica de los dientes. Su introducción formal en ortodoncia se produjo en la década de los 80 cuando Sheridan comenzó a publicar sobre este tema. Sin embargo, durante muchos años ha sido realizado en forma empírica por aquellos ortodoncistas que lo practicaban mientras que la mayoría de los dentistas lo desechaba sin mayor consideración.

La técnica de reducción de esmalte interproximal, no ha sido muy bien vista hasta ahora porque se creía que podía favorecer la aparición de caries, al pro- ducir un alto grado de desmineralización. Varios estudios realizados por prestigiosos cariólogos, han demostrado que el desgaste inicial del esmalte provoca una reacción de defensa en el diente, en la que se crean zonas de nucleación para una remineralización acelerada. A los pocos minutos la saliva está neutralizando las zonas desgastadas y el proceso de remineralización y fortaleza del esmalte puede empezar en una hora. Al principio es muy rápido, pero va ralentizándose con el tiempo y en un período de unos nueve meses esta reacción se completa y el esmalte es tan resistente a la caries como antes ${ }^{2.3 . *}$.

Esta reacción tiene una explicación antropológica ${ }^{4}$. Hace millones de años, nuestros antepasados utilizaban los dientes además de para masticar los alimentos, como un arma y una herramienta para moler, afilar, etc. Esto provocaba un desgaste muy grande de las superficies oclusales e interproximales, que originó un mecanismo de adaptación a través del tiempo. Esta adaptación estructural, favoreció la formación de una mayor cantidad de esmalte más duro, en esas zonas donde se producía el desgaste. Además se creó un sistema de remineralización acelerada de esas zonas y una erupción continua para mantener la dimensión vertical y preservar una buena función muscular. Así mismo, se estableció la formación continuada de dentina secundaria, para ir alejando la pulpa de esta atrición y la presencia de dolor en el caso de que este desgaste fuese más rápido que el efecto de los sistemas reparadores.

Las cosas empezaron a cambiar con la utilización del fuego y de las herramientas, que han hecho perder la hegemonía funcional al sistema masticatorio en aras de una mayor importancia social. La naturaleza no ha borrado estos millones de años de evolución y seguimos teniendo el mismo sistema reparador de la atrición, ya que no interfiere con la habilidad de reproducción ni de prolongación de la vida que serían razones poderosas para modificarlas. Otros ejemplos parecidos son las unas de las manos y los pies y el crecimiento continuo del pelo, así como la presencia de una capa gruesa de esmalte en las zonas interproximales. Estos tejidos que son vestigios del pasado, no sirven de gran ayuda y en el caso del esmalte, muchas veces ocupa espacios que se necesitan para resolver los problemas de apiñamiento dental ${ }^{4}$.

\section{Distancia intercadicular u su relación con defectos periodontales}

Si bien es sabido que los defectos periodontales son el resultado del balance entre el efecto de la placa bacteriana acumulada en la bolsa periodontal y los factores de riesgo del paciente en particular, es cierto que la topografía del defecto generado está condicionada por una variedad de factores existentes. Así se han descrito los efectos que la extensión de la inflamación, el trauma oclusal, la morfología del proceso alveolar, la posición y anatomía dental, la relación de las crestas marginales contiguas, la posición de los límites amelocementarios vecinos o los puntos de contacto abiertos, puedan tener en el tipo de defecto que se produzca ${ }^{5-8}$. 
Otro de los factores influyentes en la creación de defectos, es la distancia interradicular. Un espacio estrecho representa un factor negativo en pacientes con enfermedad periodontal, ya que puede impedir la limpieza, alterar el contorno gingival, complicar el tratamiento y afectar la vulnerabilidad del septum óseo. Así, dientes apiñados han demostrado mayor predisposición a la enfermedad periodontal?.

La distancia interradicular influye también en el tipo de pérdida ósea que se pueda presentar, a menor distancia entre las raíces, es menor la prevalencia de defectos intraóseos en dientes alineados y con buena relación de contacto ${ }^{10}$. Se ha demostrado que el porcentaje de defectos intraóseos aumenta conforme lo hace la distancia entre las raíces. El mayor porcentaje se encontró cuando la separación de las raíces variaba entre 2,5 y $4,5 \mathrm{~mm}^{11}$. Esto concuerda con el hallazgo de Waerhaug de que el radio de acción de la placa dental es de alrededor de $2,5 \mathrm{~mm}^{12}$.

\section{Creación de espacios}

Los dientes se alinean en un mismo arco relacionados por las zonas de contacto. Éstas conforman las troneras que alojan a las papilas interdentarias. En condiciones de salud periodontal la papila ocupa totalmente ese espacio adaptándose a filo de cuchillo con un margen delgado terminando en forma piramidal inmediatamente apical a la relación de contacto. En presencia de inflamación, el tejido aumenta de volumen, cambia su textura y llega a sangrar con facilidad. La tronera "queda pequeña" como para

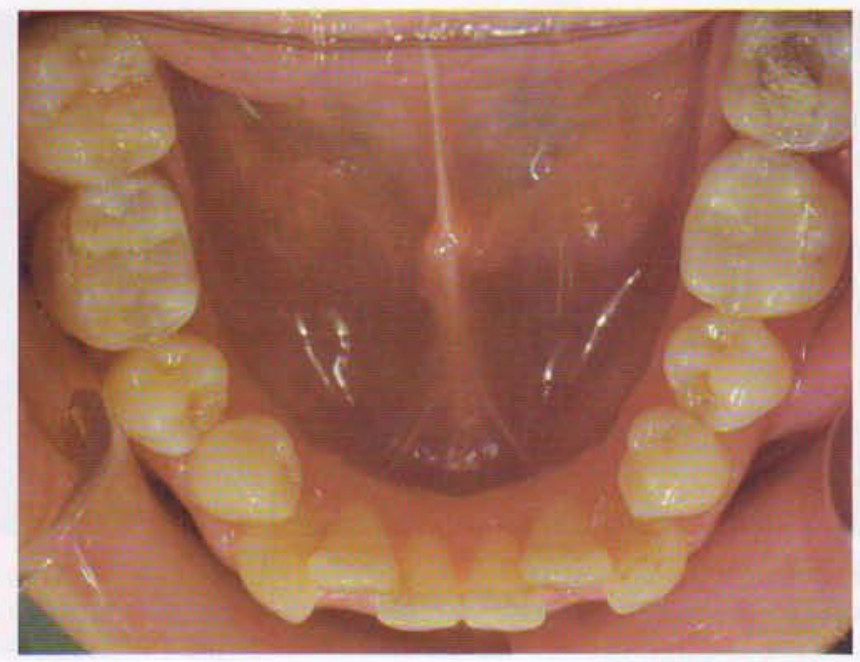

Figura 1-A. Situación preoperatoria de la arcada inferior.

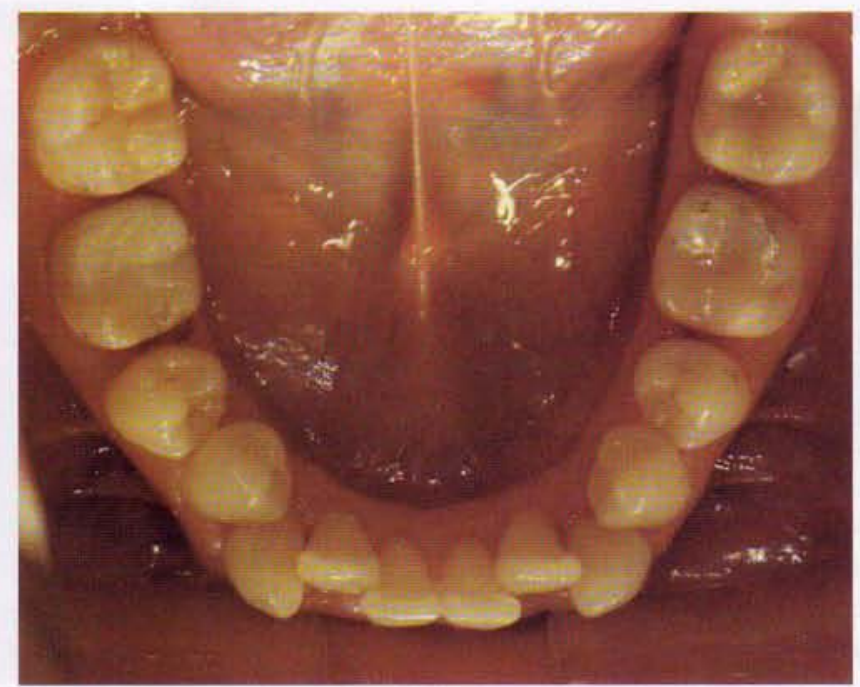

Figura 1-B. Primeros desgastes entre los dientes más distales.

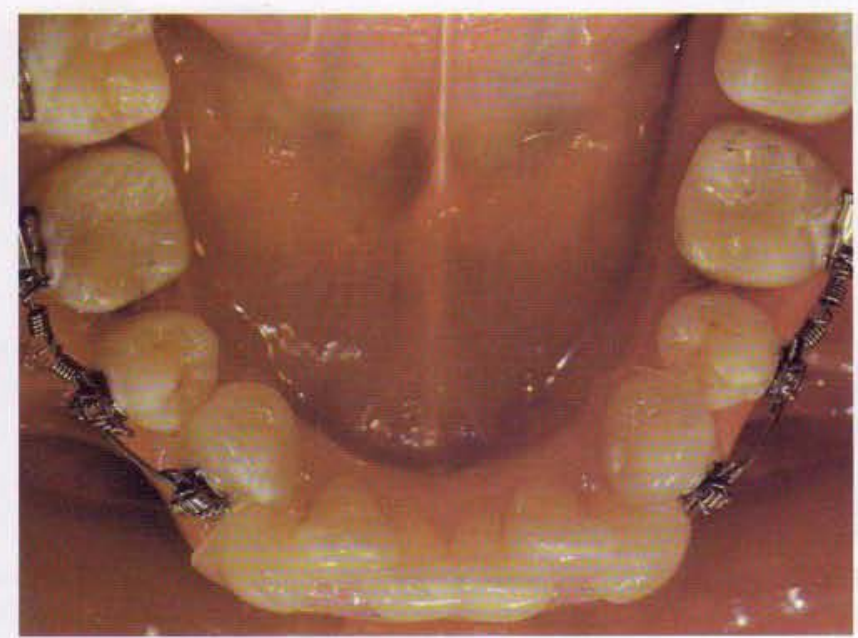

Figura 1-C. Distalización de los primeros molares con resortes por medio de aparatología seccionada. 


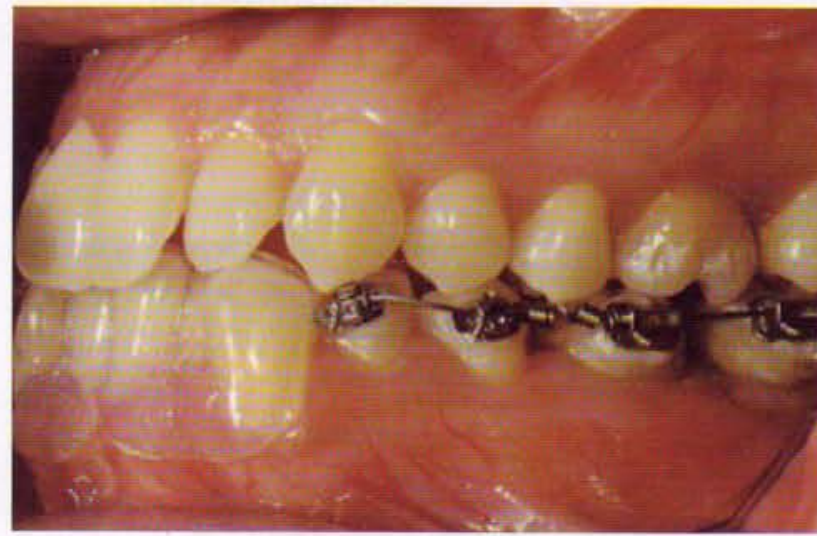

Figura 1-D. Utilizando una férula de contención removible se previene la inclinación hacia delante de los incisivos.

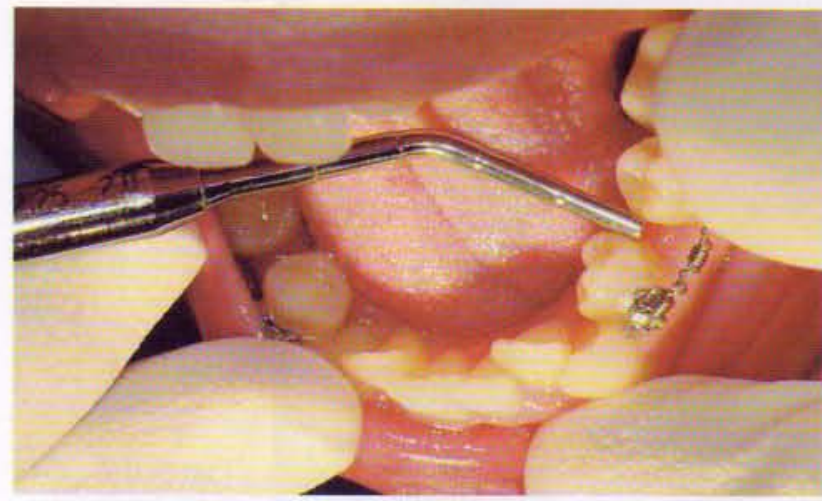

Figura 1-F. Previo al desgaste, se mide el espacio existente con la guía espacial de Sheridan. En el mango se observan los valores que corresponden a los diferentes grosores de la guia.

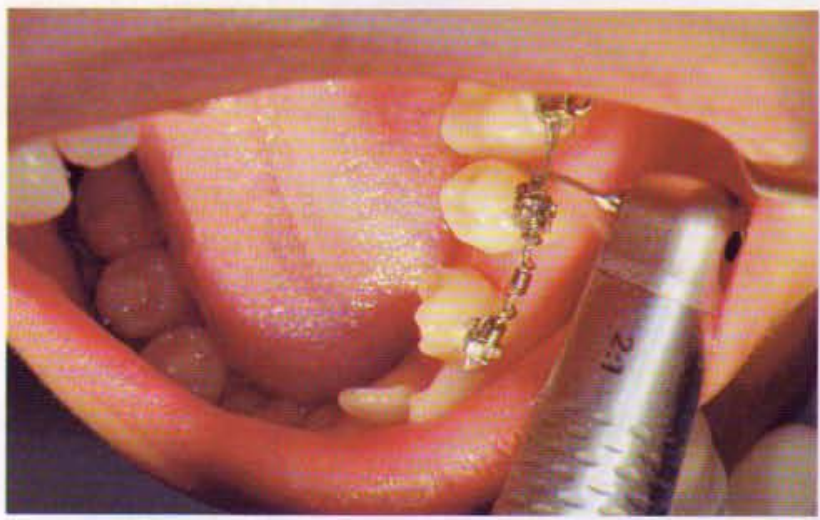

Figura 1-H. Con limas del sistema EVA, se puede pulir el remodelado y redondear zonas de dificil acceso.

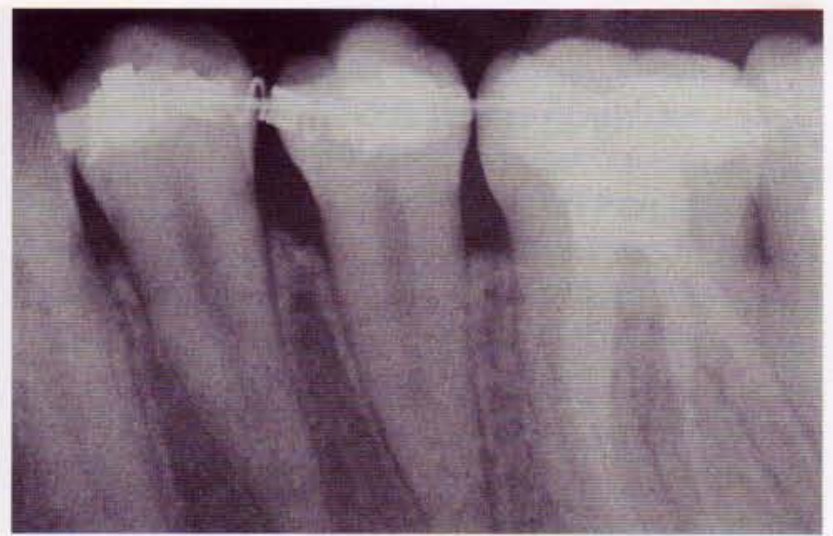

Figura 1-E. La radiografia muestra el desgaste realizado en mesial del primer molar y en distal del segundo premolar. La cara mesial aún no ha sido desgastada. Con el desgaste realizado se ha conseguir bastante espacio con una mínima reducción de esmalte.

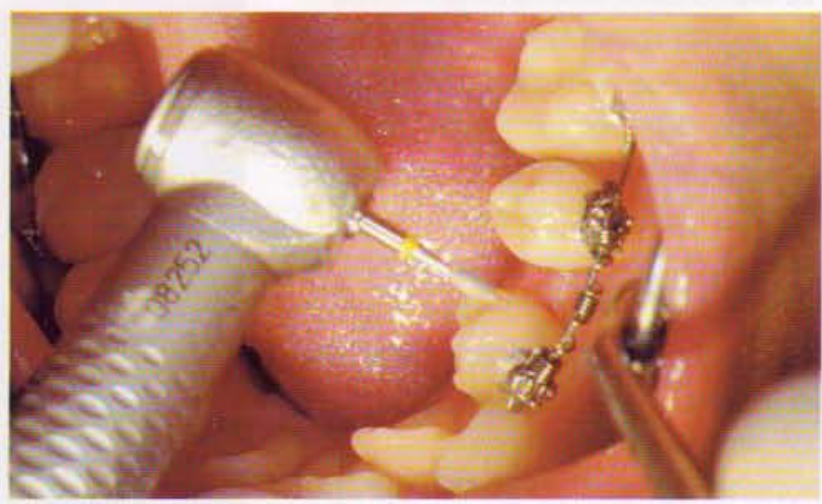

Figura l-G. Realización del remodelado interproximal con una fresa de diamante de grano fino.

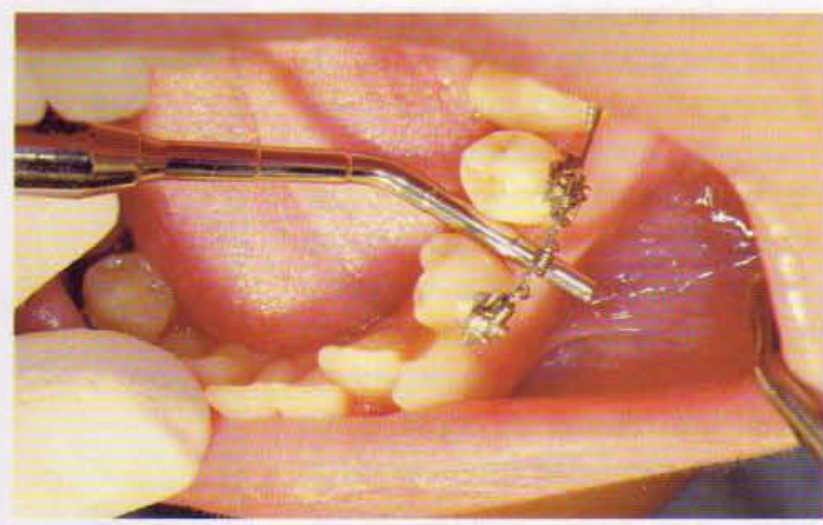

Figura 1-I. Midiendo el espacio después del desgaste. Se puede saber en cada momento cuánto esmalte se ha desgastado utilizando esta guía. 


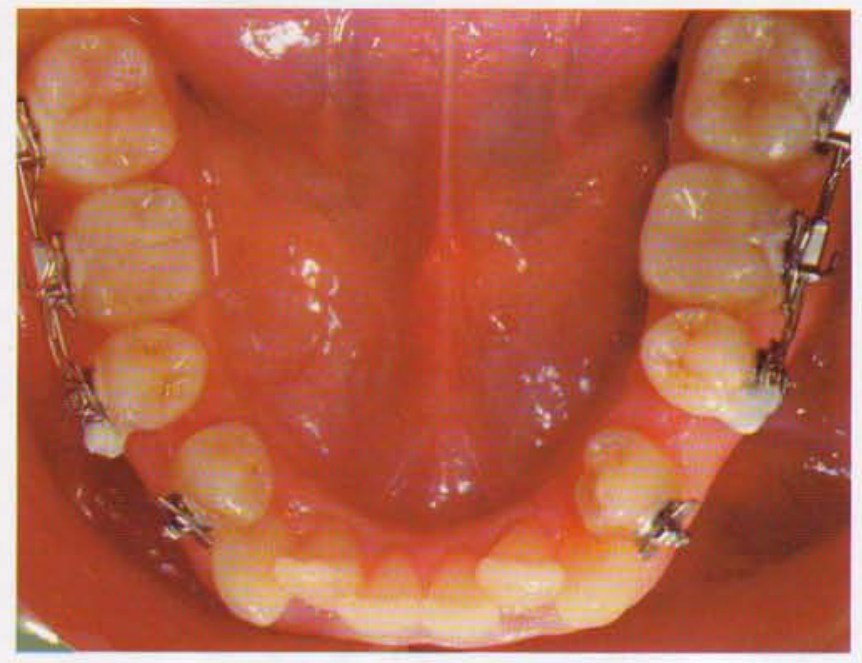

Fig 1-J. Los sectores posteriores bilaterales ya están distalizados y estabilizados. Se observan también los espacios ganados en ambos lados que se utilizarán para corregir el apiñamiento anterior.
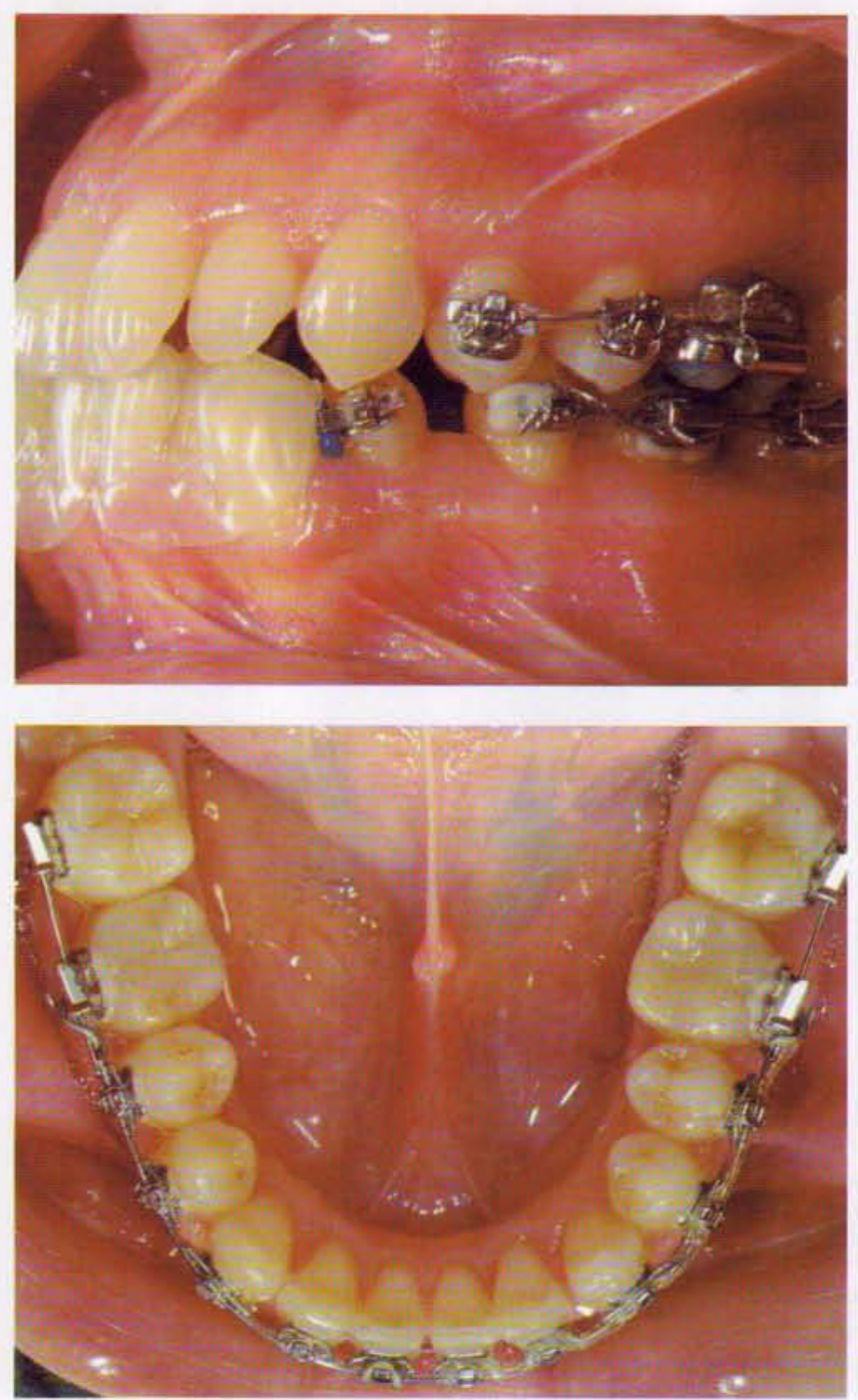
parando esta imagen con la fig I-D, se aprecia que se ha ganado el espacio distalizando molares $y$ premolares, pero no inclinando los incisivos hacia delante.

Fig 1-L. Resultado logrado después de repartir los espacios que han permitido la alineación del sector anterior.
Fig 1-K. Com-

alojar a la papila inflamada. En presencia de pérdida de soporte periodontal, sin embargo, los tejidos pierden inserción al diente, el hueso se reabsorbe y la tronera aumenta su tamaño. Controlada la inflamación, existe ahora ante la presencia de pérdida de soporte periodontal, espacio más que suficiente para albergar la papila.

Cuando se tiene que tratar un problema ortodóncico en un adulto en el que falta espacio para poder alinear y nivelar los dientes, se tienen dos opciones: aumentar el perímetro de las arcadas mediante expansión e inclinación hacia delante, o reducir el espacio que ocupan los dientes. La primera no es muy atractiva periodontalmente porque lleva los dientes hacia las corticales creando dehiscencias óseas y favoreciendo la pérdida de encía adherida. La segunda tiene dos formas de hacerse; mediante la extracción de algún diente o utilizando la técnica de reducción de esmalte. Muchas veces es inevitable hacer extracciones para conseguir los objetivos ortodóncicos, pero esta opción puede no ser tan beneficiosa periodontalmente. Con la extracción de uno, dos o cuatro dientes, se crean los correspondientes defectos óseos que en el adulto suelen permanecer de forma residual después del tratamiento ortodóncico lesta complicación es menos frecuente en niños). Por otra parte, existe la posibilidad de que al cerrar los espacios, los dientes involucrados se inclinen, alterando la relación de paralelismo entre las raíces vecinas ${ }^{6}$. Al mismo tiempo, la distancia interradicular, puede no verse reducida adecuadamente creándose triángulos negros o diastemas ${ }^{13,14}$. Aunque no evaluado en dientes sino con implantes, Tarnow y colaboradores han 
Figura 2. Caso 1 .
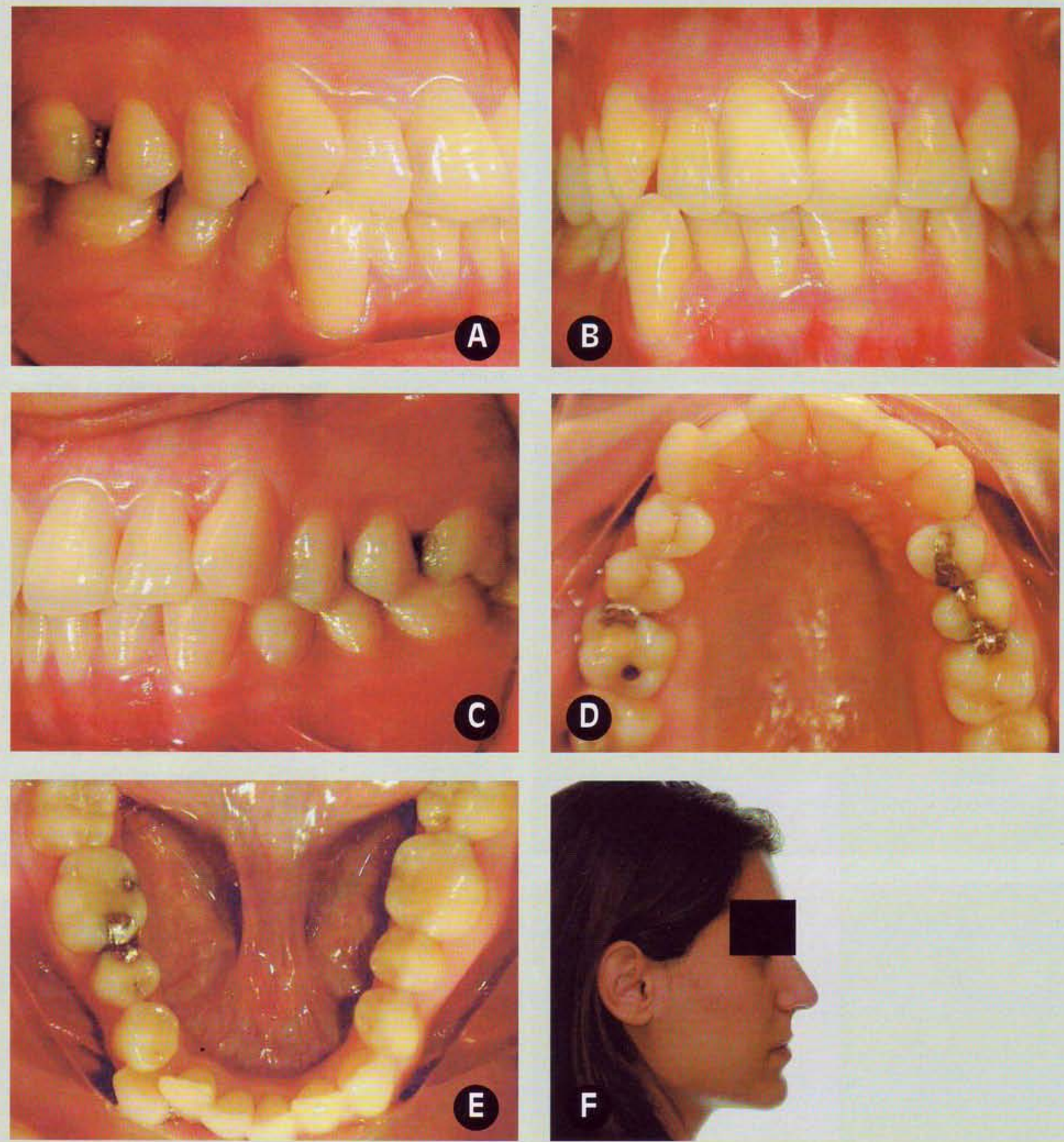

Paciente de sexo femenino, de 28 años de edad, periodontalmente sana con maloclusión y apiñamiento severo del sector anterior superior e inferior. Las figuras 2 A-E muestran la condición preoperatoria. Nótese la severa vestibuloversión del canino inferior derecho, que está en mordida cruzada. Ya que la paciente presentaba salud periodontal, el tratamiento que recibió fue de profilaxis preventiva que continuó rutinariamente durante el tratamiento ortodóncico. La figura 2-F muestra el perfil de la paciente. De haberse tratado su maloclusión con extracciones, se hubiera alterado negativamente su perfil, por lo cual se decidió ganar espacio por medio del «stripping». 

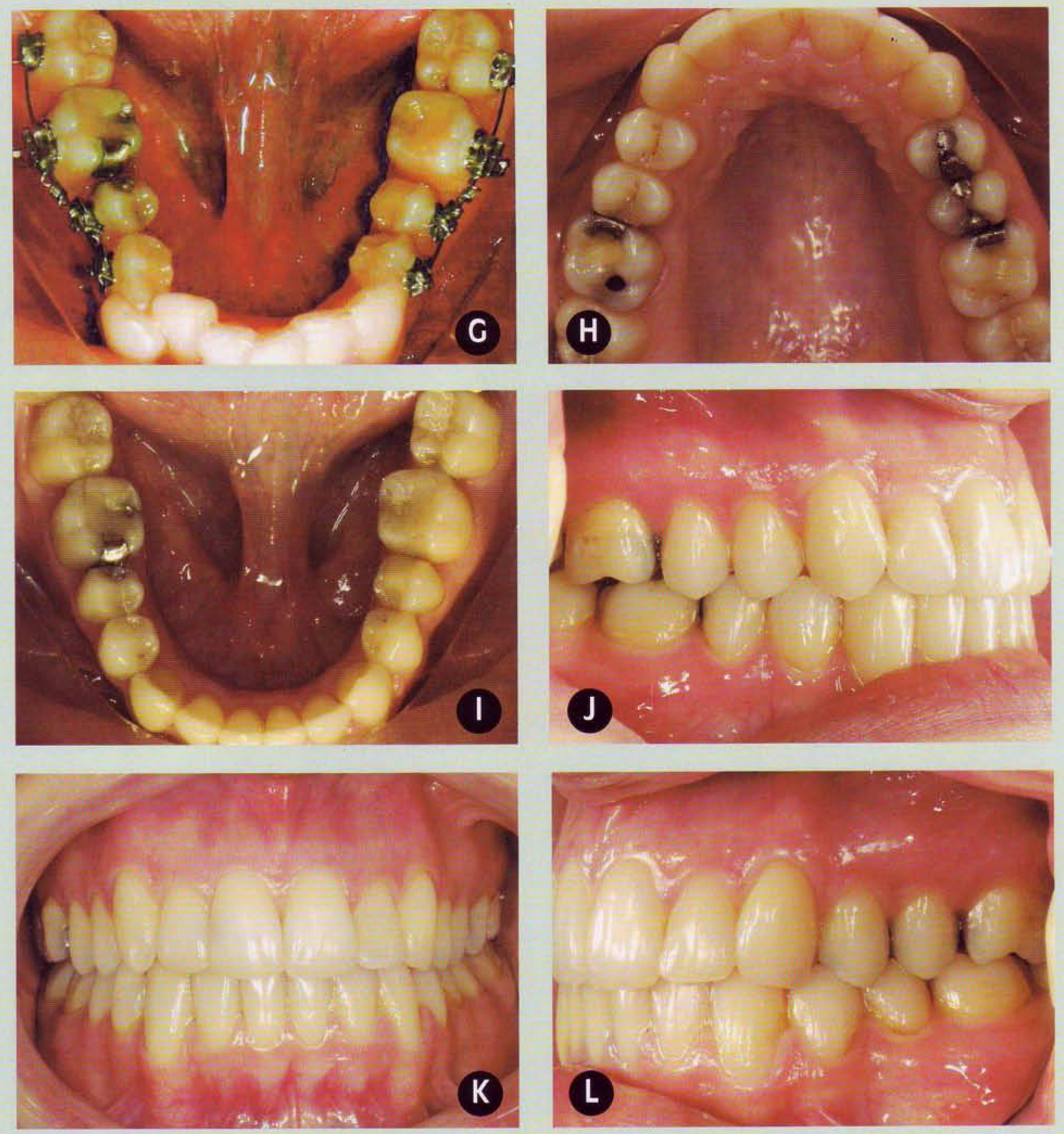

La figura 2-G muestra una etapa intermedia del movimiento ortodóncico. Se han distalizado los primeros molares observándose el espacio en mesial de los mismos. Las figuras $2 \mathrm{H}-\mathrm{L}$ presentan la situación postoperatoria. Se ha logrado la corrección de la maloclusión con la ubicación correcta de todo el sector anterior. Por medio del "stripping» se crearon 8 $\mathrm{mm}$ de espacio. El tiempo de tratamiento fue de 19 meses. 
Figura 3. Caso 2.
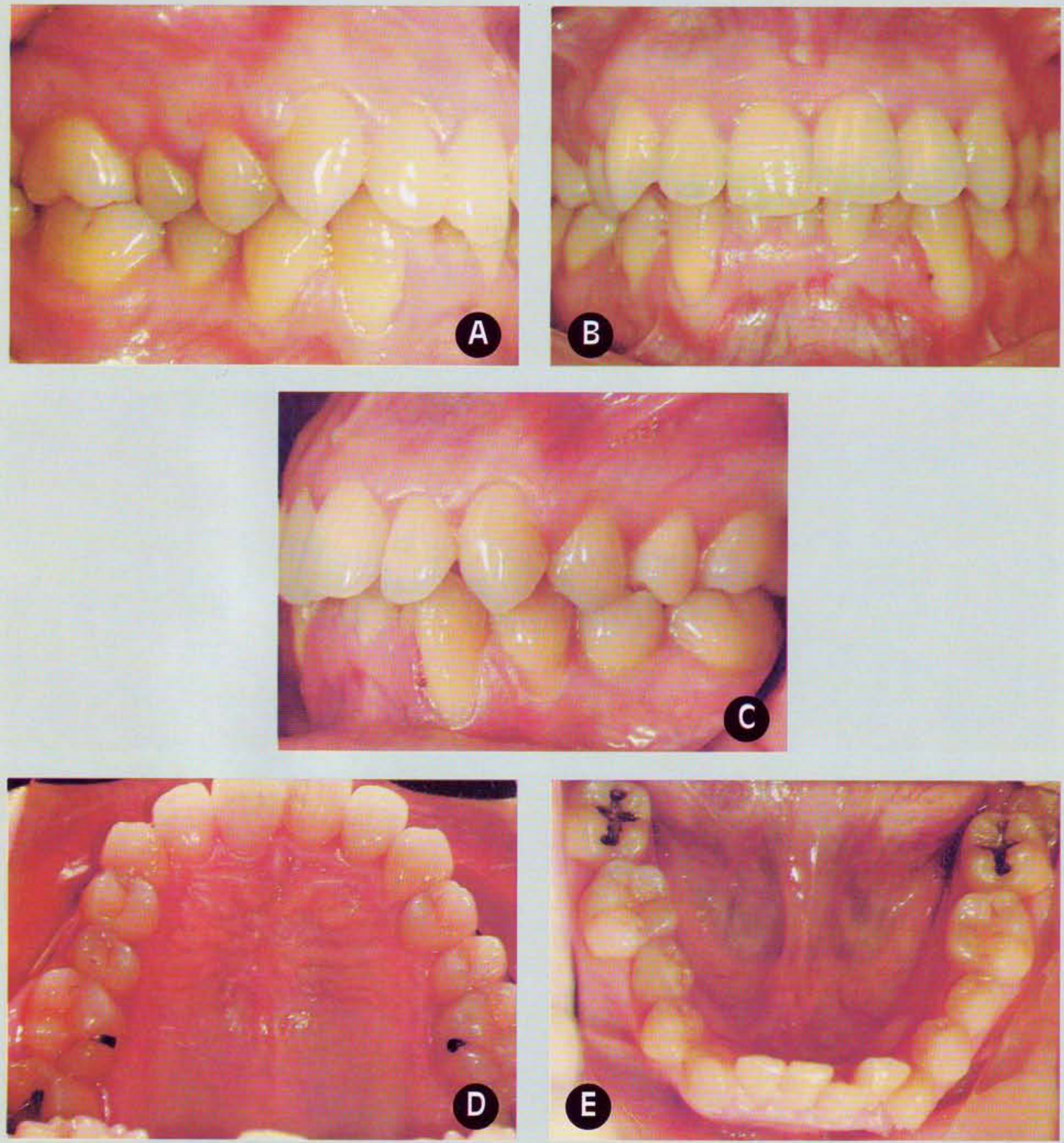

Paciente de sexo masculino, de 34 años de edad, con periodontitis crónica moderada, maloclusión y apiñamiento severo del sector antero inferior. Las figuras 3 A-E muestran la situación preoperatoria. El paciente no aceptó tratamiento ortodóncico debido a que varios especialistas recomendaban bien la extracción de cuatro premolares o un incisivo inferior, para realizarlo. Tres años más tarde el paciente decidió comenzar tratamiento ortodóncico, al indicársele que el espacio necesario sería ganado por medio de «stripping». Las figuras anteriores muestran el estado clínico antes de iniciar tratamiento alguno. 

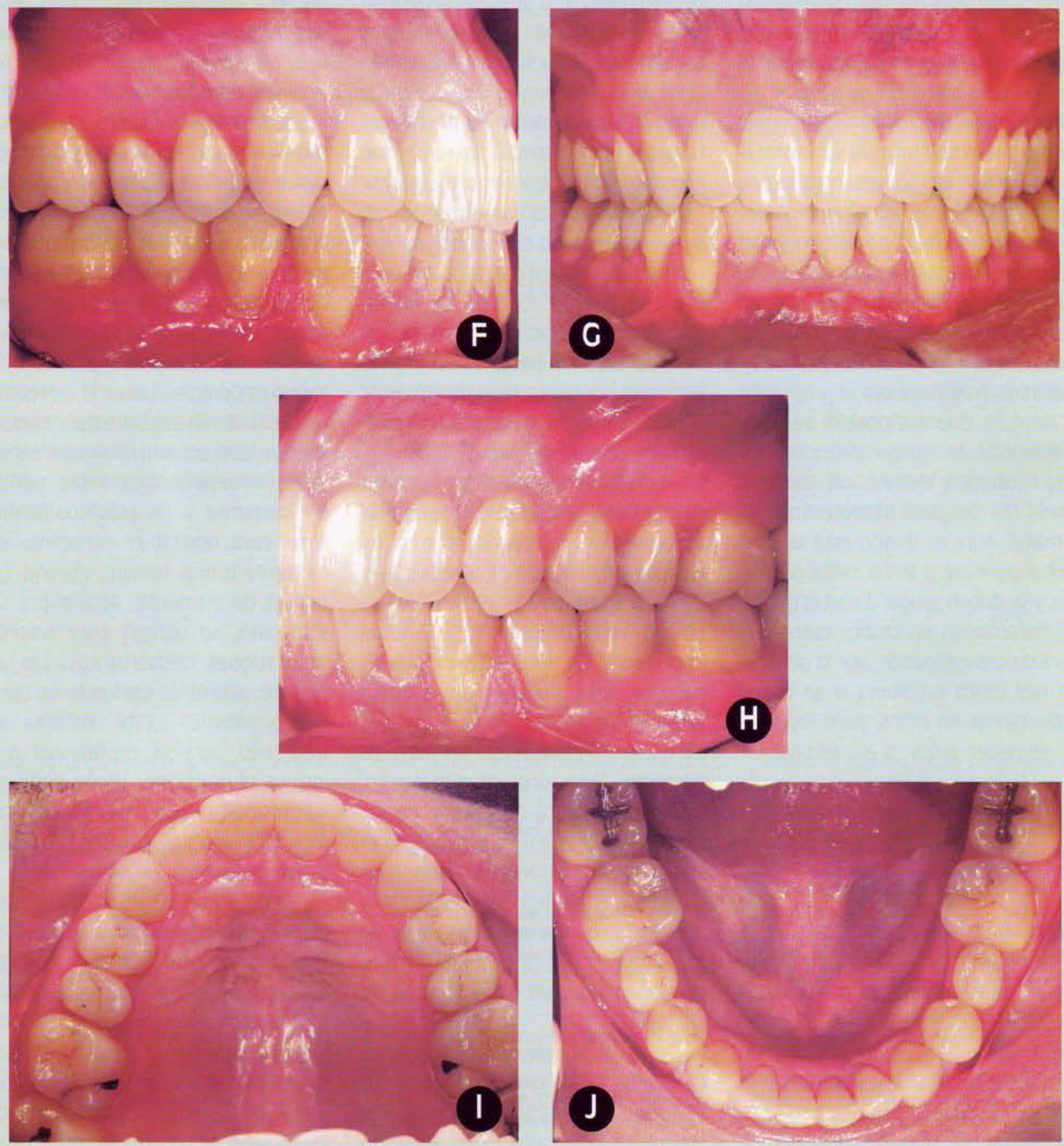

El paciente fue remitido para evaluación y terapia periodontal. Completada ésta con técnicas no quirúrgicas, el movimiento ortodóncico fue iniciado. El tiempo total fue de 18 meses y se obtuvieron alrededor de 7 mm para solucionar el apiñamiento inferior sin extracciones. Las figuras $3 \mathrm{~F}-J$ muestran el resultado logrado al completar el tratamiento, donde se puede observar el alineamiento del sector anteroinferior asi como el obtenido en el arco superior. 
demostrado que la distancia ideal entre superficies vecinas debe ser de alrededor de $3 \mathrm{~mm}^{14}$. Con las extracciones no se puede elegir la cantidad de espacio a ganar ya que hay que eliminar todo el diente; es todo o nada. De este modo en muchas oportunidades se pasa de no tener suficiente espacio a tenerlo en exceso que puede ser aún más complicado de solucionar. Además, el tratamiento ortodóncico se hace más largo, los pacientes pueden rechazarlo porque no quieren perder más dientes y generalmente el tratamiento es más complejo.

Siempre que sea posible, se debe contemplar la opción de conseguir esta reducción del espacio dental a través del desgaste interproximal del esmalte. Aquí es donde está la diferencia principal al tratar niños o adultos, y se deben aplicar distintas pautas de tratamiento en ambos casos a la hora de crear espacios. Por lo general, no hay tanto problema si se hacen extracciones en niños, pero en adultos se deben evitar, si ello es posible.

\section{Ventajas 丩 lécrica del «stripping"}

La técnica de "stripping" permite regular precisamente la cantidad de espacio a obtener para solucionar el apiñamiento. La realización de un remontaje en cera de los dientes ayuda a determinar la posibilidad de utilizar esta técnica, la cantidad de esmalte a desgastar y la localización del mismo.

Al desgastar las coronas, es posible reducir la distancia interradicular y desplazar la relación de contacto interproximal en sentido apical. Esto permite reducir, o eliminar, la posibilidad de creación de triángulos negros después del tratamiento periodontal. Comparado con un enfoque extraccionista, el tratamiento es más rápido, favoreciendo aún más la resolución efectiva del problema ortodóncico. De modo que esta técnica de "stripping" realizada en el paciente adulto, no afecta negativamente ni al diente ni a sus tejidos de soporte ${ }^{15}$. Su utilización hace más factible que tanto el dentista como el paciente acepten la incorporación del tratamiento ortodóncico como parte de la terapia multicisciplinaria en casos complejos.

Ahora bien, todo lo dicho anteriormente tiene validez, sólo si se hace el desgaste con una técnica correcta y respetando una serie de pautas que el Dr. Jack Sheridan ha descrito a lo largo de varias décadas ${ }^{16^{*} \cdot 17}$. Especial importancia tiene el hecho de que las paredes más gruesas de esmalte están en los molares y premolares y es alli donde hay que hacer los mayores desgastes. Esto contrasta con la práctica más corriente de hacerlo en los incisivos ya que ahí es donde está el apiñamiento. Aplicando su método de desgastes, manejo de espacios y anclajes, se pueden llegar a solucionar sin extracciones casos de hasta $8 \mathrm{~mm}$ de apiñamiento sin dañar la salud de los dientes.

Después de evaluar clínica y radiográficamente los dientes a desgastar se debe comenzar obteniendo el espacio entre el primero y el segundo molar. Se colocará un separador y se comenzará a desgastar las caras proximales de ese espacio con una fresa de diamante muy delgada de grano fino a alta velocidad. El esmalte debe ser "pincelado" desde cervical a oclusal bajo irrigación abundante. Este desgaste elimina el punto de contacto y agranda la relación del mismo. Una vez logrado esto, se procede a redondear los desgastes para lograr que la zona instrumentada continúe suavemente con el contorno normal del diente. Esto se logra utilizando una punta de diamante fina en forma de pera. El área debe estar perfectamente pulida y se recomienda la aplicación de flúor. Los desgastes pueden también iniciarse y pulirse utilizando el Complex-Lloret W \& $\mathrm{H}$ (sistema EVA). Es una cabeza para contraángulo que genera movimientos de vaivén. Puede activar limas, puntas de diamante y de plástico. Existen limas para realizar el "stripping" de diferente forma, tamaño y grano. Las puntas de diamante, activas por un solo lado, se utilizan para corregir obturaciones desbordantes. Las de plástico, son útiles para pulir las caras interproximales. Este sistema es necesario para el profesional que incluya el "stripping" como parte de su enfoque terapéutico. No es recomendable realizar los desgastes utilizando un disco.

El espacio a crear, se puede controlar utilizando la guía espacial de Sheridan que permite medir distancias que van de 0,75 a $3,0 \mathrm{~mm}$. Después de colocar una férula de contención anterior (Essix), se comienza a desplazar el primer molar distalmente por medio de un arco seccionado. Cuando los molares se han puesto en contacto, los mismos se estabilizan y se procede a realizar el desgaste entre el segundo premolar y primer molar. Desplazando sucesivamente los dientes después de los desgastes corres- 
pondientes, los espacios ganados van siendo sumados y trasladados al sector anterior para solucionar el apiñamiento ${ }^{18}$.

La figura 1 demuestra los aspectos más importantes que se deben considerar al utilizar la técnica del «stripping" en adultos con gran apiñamiento.

Esta técnica básica, aquí brevemente descrita, es necesaria cuando se requiere máximo anclaje. Puede ser modificada y simplificada de acuerdo al espacio que se deba crear, localización del apiñamiento $\mathrm{y} / \mathrm{o}$ anclaje necesario. Por ejemplo, si la necesidad es mínima, todos los desgastes pueden realizarse a la vez y no es necesaria la mecánica seccional ni la férula de contención.

\section{Casos clínicos}

Los dos casos clínicos que se presentan ilustran el resultado que se puede lograr con la utilización de la técnica de "stripping" durante el tratamiento ortodóncico de pacientes adultos periodontalmente sanos, 0 con enfermedad periodontal.

\section{Conclusiones}

Basado en la información presentada y en los casos clínicos comentados, es posible concluir lo siguiente:

1. El enfoque a seguir en el tratamiento del apinamiento debe ser diferente al considerar niños, adoles- centes 0 adultos con normalidad periodontal, que en adultos afectados periodontalmente.

2. En presencia de enfermedad periodontal con pérdida de inserción y reabsorción ósea, el espacio interdental que aloja la papila aumenta y por lo tanto es posible reducir la distancia interdental sin afectar la salud periododontal.

3. Cuando se trata ortodóncicamente al paciente adulto, la técnica de "stripping" permite obtener espacio suficiente para corregir apiñamientos y alinear los dientes sin necesidad de extracciones.

4. El "stripping" realizado cuidadosamente, no afecta ni al diente ni a los tejidos periodontales.

\section{Bibliografla recomendada}

Para profundizar en la lectura de este tema, el/los autor/es considera/an interesantes los artículos que aparecen señalados del siguiente modo: *de interés ${ }^{* *}$ de especial interés.

1. Sada Garralda V, Caffesse RG. El enfoque ortodóncico en el tratamiento multidisciplinario de pacientes adultos. Su relación con periodoncia. RCOE 2003;8(6)673-684

2. Brudebold F, Tehrani A, Bakhos Y. Intraoral mineralization of abraded dental enamel. J Dental Res 1982;65:456-9

3**. El-Mangoury NH,Moussa M. In vivo remineralization after air rotor Stripping. J Clin Orthodon 1991;25:75-8.

Los resultados de este estudio establecen que la reducción de esmalte interproximal en el sector posterior, no parece exponer a los dientes a cambios patológicos que puedan llevar a caries; sino a un periodo de remineralización que puede durar nueve meses.

4. Sheridan JJ. An anthropological rationale for air-rotor stripping(ars). En Sheridan JJ:The updated air-rotor stripping (ars) manual. Raintree Essix, Inc. Capitulo 1. (Ortho Spain).

5. Macapanpan I, Weinmann JP. The influence of injury to the periodontal membrane on spread of gingival inflammation. J Dent Res 1954;33:263-72
6. Pritchard J. The effect of bicuspid extraction orthodontics on the periodontium. J Periodontol 1975;46:534-42.

7. Glickman I, Smullow JB. Alternations in the pathway of gingival inflammation into the underlying tissue including excessive occlusal forces. J Periodontol 1962;33:7-13.

8. Goldman $\mathrm{H}$, Cohen $\mathrm{DW}$. The intrabony pocket: classification and treatment. J Periodontol 1958:29:272-91.

9. Pennel B, Keagle J. Predisposing factors in the etiology of chronic inflammatory periodontal disease. J Periodontol 1977;48:517-32

10. Heins PJ, Thomas RG, Newton JW. The relationship of interradicular width and alveolar bone loss. A radiometric study of a periodontitis population. J Periodontol 1988;59:73-9.

11. Tal H. Relationship between the interproximal distance of roots and the prevalence of intrabony pockets. J Periodontol 1984;55:604-7.

12 . Werhaug $J$. The infrabony pocket and its relationship to trauma from occlusion and subgingival plaque. J Periodontol 1979;50:35565.
13. Tamow D, Magner AW, Fletcher P. The effect of distance from the contact point to the crest of bone on the presence or absence of the interproximal dental papilla. J Periodontol 1992; 63:995-6.

14. Tarnow DP, Cho SC, Wallace SS. The effect of inter-implant distance on the height of interimplant bone crest. J Periodontol 2000; 71:546-9.

15. Crain G, Sheridan JJ. The susceptibility to caries and periodontal disease after air-rotor stripping. J Clin Orthod 1990;24:84-5.

$16^{* *}$. Sheridan JJ. Air-rotor stripping. J Clin Orthod 1985;19:43-59.

En este artículo se describe la técnica de «stripping) para desgastar con precisión cantidades de esmalte de hasta $10 \mathrm{~mm}$ y el método de consolidación de esta espacios para solucionar el apiñamiento anterior.

17. Sheridan JJ. Air-rotor stripping update. J Clin Orthod 1987;21:781-8.

18. Ballard R, Sheridan JJ. Air-rotor stripping and the Essix anterior anchor. $\mathrm{J}$ Clin Orthod 1996;30:371-3. 